\title{
Uso y conocimiento de los servicios públicos loca- les por parte de la población inmigrante. La importancia del barrio de residencia
}

\author{
María Ángeles HuETE GARCÍA \\ Universidad Pablo de Olavide \\ mahuegar@upo.es
}

Recibido: 27-09-2011

Aceptado con modificaciones: 10-07-2012

Aceptado: 29-11-2012

\section{Resumen}

Este artículo analiza los patrones de uso y conocimiento de los servicios municipales por parte de la población inmigrante residente en la ciudad de Sevilla. Dicho análisis se realiza atendiendo al contexto donde residen los inmigrantes, esto es, su barrio de residencia, así como su situación de integración, en atención a tres dimensiones concretas: económica, social y cívica. Los resultados muestran que, efectivamente, el barrio de residencia puede configurar subculturas urbanas propias, en este caso, aplicadas a las formas de uso y conocimiento sobre los servicios municipales. De la misma forma, las diferentes dimensiones de la situación de integración podrían contribuir a la forma en que se usan y conocen los servicios analizados.

Palabras clave: servicios públicos locales, población inmigrante, modelo de barrio, situación de integración

\section{Use and knowledge of local public services by immigrants. The importance of the district of residence}

\begin{abstract}
This article analyzes usage patterns and knowledge of municipal services by the immigrant population residing in the city of Seville. This analysis is done taking into account the context where immigrants reside, ie, neighborhood of residence, and its position of integration, focused on three specific dimensions: economic, social and civic life. The results show that, indeed, the district of residence can set their own urban subcultures, in this case, applied to forms of use and knowledge of municipal services. Similarly, different dimensions of integration situation could contribute to the way they use and know the services analyzed.
\end{abstract}

Keywords: public local services, migrant population, model of disctrict, integration.

\section{Referencia normalizada}

Huete García, P. (2013). "Uso y conocimiento de los servicios públicos locales por parte de la población inmigrante. La importancia del barrio de residencia”. Política y Sociedad, Vol.50 núm. 1: páginas. 215-234

Sumario: Introducción. 1.Marco analítico y operacionalización. 2. Fuentes de información utilizadas. 3.La población inmigrante de Sevilla. Perfiles y lógicas de localización. 4.Patrones de interacción con los servicios públicos locales de las personas inmigrantes en la ciudad de Sevilla: sobre uso y conocimiento. 5.Conclusiones. 6.Referencias bibliográficas 


\section{Introducción}

No cabe duda que el incremento de la población inmigrante es un rasgo destacable en la configuración del perfil de la sociedad española desde hace más de una década. La llegada de nuevos vecinos a nuestras ciudades y barrios, ha supuesto no solamente el incremento de mano de obra en el mercado laboral, sino también una serie de nuevos retos para los gobiernos y responsables políticos. Dichos retos se plantean en relación con aspectos como la participación ciudadana, para lograr dar voz a los nuevos vecinos, o el acceso a los equipamientos y servicios, que han de responder a sus demandas y necesidades.

En este sentido, comienzan a plantearse, por una parte, cuestiones en relación con los patrones de participación política, bien en el ámbito formal, a través del reconocimiento del derecho a votar (Bevelander y Pendakur, 2008); o bien, facilitando su participación a través de mecanismos informales, como presupuestos participativos o foros ciudadanos, destinados explícitamente a la participación de las personas inmigrantes (González-Ferrer y Morales, 2006).

Por otra parte, la población inmigrante, dado su perfil, representa nuevos retos al Estado del Bienestar1, que se han de poner de manifiesto en su forma de acceso y uso, tanto sobre los equipamientos y servicios públicos, como de las administraciones encargadas de suministrarlos.

Este artículo aborda esta cuestión. De forma concreta, se plantea que las personas inmigrantes, por el hecho de serlo, no constituirán un patrón único en cuanto a su relación con los servicios públicos, sino que mostrarán diferencias en atención a aspectos relacionados con el contexto en el que viven, concretamente el barrio de residencia, así como sus características o recursos individuales, analizado en este trabajo en relación su situación de integración en la sociedad de acogida.

Para ello, el artículo se estructura de la siguiente forma. La primera sección se destina a la elaboración del marco analítico y su operacionalización, donde se plantean las principales hipótesis sobre la relación entre el modelo de barrio y la situación de integración del individuo y los patrones de uso y conocimiento de los servicios municipales. La segunda sección se destina a la descripción de las fuentes de información que se utilizan en los análisis destinados al contraste de las hipótesis. La tercera sección ofrece información general sobre el proceso migratorio de la ciudad que constituye el caso de estudio, Sevilla, para en la sección cuarta mostrar los análisis sobre los patrones de uso y conocimiento sobre los servicios públicos locales por parte de la población inmigrante. La última sección se destina a las conclusiones.

\section{Marco analítico y operacionalización}

Esta sección se destina a ofrecer información sobre las hipótesis planteadas, así como su transformación en medidas concretas a través del proceso de operacionalización. Tal como se ha señalado al inicio del artículo, este trabajo se destina a 
conocer las formas de interacción de la población inmigrante con el sector público, a través de dos cuestiones concretas, como son, su grado de conocimiento y patrones de uso de determinados servicios públicos municipales². Dichos aspectos se consideran básicos a la hora de analizar la relación de la ciudadanía con los servicios públicos. Por una parte, el conocimiento sobre la existencia de los mismos es un elemento básico y de partida a considerar a la hora de analizar el uso y valoración de la ciudadanía otorga a los servicios, pues solamente aquellos que los ciudadanos saben que tienen a su disposición se pueden utilizar y, en su caso, valorar. De esta forma, partir del conocimiento de los servicios se consideraba un aspecto básico a la hora de conocer los patrones de interacción de la población inmigrante con los servicios públicos.

Por otra parte, es necesario realizar una breve aclaración en relación con la tipología de servicios utilizada. Si bien existen en la literatura múltiples tipologías en relación con los servicios (Stein, 1990; Buchanam, 1971; Peterson, 1982), este trabajo establece una única diferenciación entre servicios específicos y no específicos o de carácter comunitario. Dicha diferenciación se realiza a partir de la literatura sobre servicios sociales, en la que habitualmente se distingue entre servicios de carácter general y servicios de carácter específico. De esta forma, por ejemplo Hidalgo (1985), en su análisis sobre los servicios sociales en Andalucía a partir de la normativa que los regula, diferencia entre servicios sociales de carácter general, destinados a la sociedad en su conjunto y servicios sociales de carácter específico, donde se incluyen de forma explícita "servicios de protección al emigrante" (1985:213).

En este sentido, se asume que la naturaleza de los servicios, en este caso más o menos orientados a la población inmigrante, podría relacionarse de forma distinta con los factores que hemos considerado explicativos de los niveles de uso y conocimiento de los servicios, al constituir no solamente oportunidades concretas en términos de qué bienes y servicios prestan a los individuos, sino también diferentes grados de integración de los individuos, dados los requisitos necesarios para su utilización.

Dicha diferenciación teórica se pone en evidencia además en el análisis factorial realizado con los datos de la encuesta con la que se trabaja, pues se pone de manifiesto la existencia de dos dimensiones. Por una parte, aquellos servicios destinados específicamente a la atención de las personas inmigrantes, como son el soporte de atención a las personas inmigrantes (SOMAI) y el foro de participación de personas inmigrantes; por otra parte, servicios de carácter comunitario, destinados a todos los ciudadanos con independencia de su origen geográfico, como son servicios destinados al fomento del empleo (programa REDES y Bonobús Solidario), servicios sociales básicos (unidades de trabajo social), instalaciones deportivas básicas municipales o mecanismos de participación destinados a la población en su conjunto, como son los presupuestos participativos. 
Tabla 1. Matriz de componentes ${ }^{\mathrm{a}}$

\begin{tabular}{|l|c|c|}
\hline \multirow{2}{*}{} & \multicolumn{2}{|c|}{ Componente } \\
\cline { 2 - 3 } Soporte Mínimo de Atención a los Inmigrantes & 1 & 2 \\
\hline Consejo municipal de participación del migrante &, 442 & 0,762 \\
\hline Instalaciones deportivas básicas municipales & 0,601 &,- 335 \\
\hline Presupuestos participativos & 0,510 &, 393 \\
\hline Programa REDES & 0,771 &,- 257 \\
\hline Bonobús solidario & 0,712 &,- 220 \\
\hline Unidades de trabajo social & 0,686 &,- 411 \\
\hline Método de extracción: Análisis de componentes principales. \\
\hline a. 2 componentes extraídos
\end{tabular}

En atención al contexto o barrio de residencia, este trabajo analiza diferentes modelos de barrio como contexto propio para la generación de patrones de uso y conocimiento sobre los servicios de la población inmigrante. Dichos modelos se crean en función de dos dimensiones: la presencia de población inmigrante en el barrio o, dicho de otra forma, el volumen del contingente inmigrante y el grado de diversidad de las nacionalidades presentes en el mismo.

Del cruce de ambas dimensiones, surgen cuatro modelos de barrio. A saber: barrios con alta presencia inmigrante pero baja heterogeneidad de nacionalidades, que representarían casos extremos de segregación. Por el contrario, barrios con alta presencia inmigrante y alta heterogeneidad, que estarían reflejando situaciones de multi o interculturalidad. Entre ambas situaciones, se encontrarían barrios con baja presencia de población inmigrante y poca heterogeneidad, que podríamos denominar autóctonos. Y finalmente barrios, que podríamos denominar en auge, con baja presencia inmigrante pero en los que en esa baja presencia se daría una gran variedad de nacionalidades.

Ilustración 1. Modelos de barrio en atención a presencia y diversidad.

\begin{tabular}{|c|l|r|}
\hline \multirow{2}{*}{} & \multicolumn{2}{|c|}{ Presencia } \\
\cline { 2 - 3 } Diversidad & Multicultural & En auge \\
\cline { 2 - 3 } & & \\
\cline { 2 - 3 } & Segregado & Autóctono \\
\hline
\end{tabular}


Es importante señalar que, para el caso de Sevilla, tras los análisis realizados no se dieron en la realidad modelos de barrio del tipo segregado, por lo que los análisis se realizan atendiendo a los tres modelos de barrio restantes, que sí se dan en la realidad.

La hipótesis que subyace a la elaboración de dicha tipología es que la conformación sociodemográfica de los barrios, de forma concreta, el origen geográfico y diversidad de sus habitantes, deberá a su vez verse reflejado en lo que Fischer $(1975,1995)$ denominaría subculturas o subsistemas sociales, definidos a partir de creencias, valores, normas y costumbres asociadas a ellas, que para el caso de este artículo se reflejarían en los patrones de uso y conocimiento sobre los servicios públicos locales. De forma concreta, las hipótesis de Fisher se fundamentan sobre la base de tres proposiciones, que trataremos a aplicar al análisis de los patrones de uso de los servicios en el marco de este trabajo. Son las siguientes:

Proposición 1. Las ciudades, por el tamaño de población que en ellas se concentra, tienen mayores posibilidades de acoger a su vez mayor número de subculturas. De forma concreta, a medida que sean más grandes en términos de población tendrán a su vez mayores posibilidades de acoger individuos procedentes de lugares diversos, y con ello con culturas propias. En este sentido, se generará además mayor diversidad en aspectos económicos, espaciales, institucionales y culturales. De esta forma, las ciudades tendrán más posibilidades de acoger subculturas diferenciadas, organizadas alrededor de costumbres, ocupaciones, ocio, etc.

Tomando en consideración esta proposición, su aplicación a este trabajo se basaría en que entendiendo que cada barrio, dado su perfil en términos de presencia y diversidad de la población inmigrante, constituye un contexto propio para el surgimiento y desarrollo de subculturas propias. En este sentido, los patrones de conocimiento y uso de los servicios públicos locales variará entre los diferentes modelos de barrio o, dicho de otra forma, el modelo de barrio ha de ser un elemento diferenciador en los patrones de uso y conocimiento de los servicios municipales entre los individuos analizados.

Proposición 2. Las ciudades no solamente son el contexto idóneo para el surgimiento de subculturas, sino que también lo son para el aumento de su intensidad. Dicha intensidad se concibe como la medida en que estas subculturas se encuentran institucionalizadas y existen entre sus miembros interacciones estables. De forma concreta, a medida que aumente el tamaño de la población se darán más subculturas que promuevan instituciones y desarrollen redes de relación entre sus miembros. Esta proposición, por tanto, parte de la base de que la concentración en el espacio generaría más posibilidades de interacción entre los individuos, comunicarse y crear lo que en términos de Durkheim podríamos llamar "densidad moral”.

En relación con la aplicación de esta proposición a nuestro artículo, la coincidencia en el espacio de personas inmigrantes, esto es, mayor presencia debería redundar en un mayor uso y conocimiento de los servicios locales, pues existirían 
mayores posibilidades de acceso a los mismos a través de facilitarse información entre unos individuos y otros en relación con su existencia y formas de uso.

Proposición 3. Las ciudades, además de ser el contexto idóneo para el surgimiento de culturas, suponen mayores posibilidades de relaciones entre los grupos, y como consecuencia, mayores posibilidades de influencia entre ellos. Dicha influencia podrá darse en dos sentidos; grupos mayoritarios sobre grupos minoritarios o bien, grupos minoritarios influyendo en los mayoritarios. En este sentido, para el caso que nos ocupa, las diferencias fundamentales se encontrarían entre los barrios autóctonos, como efecto de la presencia masiva de población autóctona, respecto a los multiculturales, como efecto de la presencia masiva de población inmigrante.

En definitiva, asumir la teoría de las subculturas urbanas supone abordar el análisis desde una perspectiva ecológica, pues considera el lugar o ámbito de residencia del individuo como elemento con capacidad para explicar su comportamiento, si bien, dicho espacio o entorno se construye a partir de los atributos (en este caso la presencia y diversidad de orígenes) de sus residentes3. Dichos subsistemas o subculturas se entenderían, en el contexto de este artículo, como creencias, valores y costumbres asociadas al uso y conocimiento de los servicios públicos locales.

Junto al barrio como contexto, este trabajo considera que la situación de integración del individuo podría introducir diferencias en el uso y conocimiento de los servicios públicos locales. Tradicionalmente, cuando se analiza el grado de integración social de las personas inmigrantes, se hace referencia no solamente a aspectos fundamentalmente relacionados con su situación económica, entendiendo que dicha integración no pasa fundamentalmente por el logro de su inserción laboral. Junto a ello, es necesario considerar otros aspectos como la interacción de las personas inmigrantes con individuos no necesariamente procedentes de su país de origen, o el hecho de tener una participación activa en la toma de decisiones en la sociedad de acogida. Dicha afirmación se basa en la premisa, ampliamente aceptada en la literatura, que por exclusión social ha de entenderse "un proceso social de pérdida de integración que incluye no solo la falta de ingresos y alejamiento del mercado laboral, sino también in descenso de la participación social y por tanto una pérdida de derechos sociales" (VVAA, 2007:27).

En este sentido, son diversos los intentos de medición del concepto de exclusión social (Commins, 1993; Laparra, 2001; García, 2010; Secretaría de Estado de Inmigración y Emigración del Ministerio de Trabajo y Asuntos Sociales, 2010), ahora bien, en todos ellos se tienen en cuenta las tres dimensiones consideradas en el artículo, a saber: los derechos políticos, a través de la participación efectiva en la toma de decisiones; los derechos económicos, a través de su participación activa en el mercado laboral; y los lazos sociales, tanto a través de la familia como de la comunidad, basados en la vecindad, la etnia, la religión u otros elementos.

Tomando en consideración estas premisas, para este trabajo la situación de integración se ha medido en atención a tres dimensiones concretas. En primer lugar, una dimensión económica, en la que se refleja información en relación con sus 
ingresos y situación de empleo; en segundo lugar, una dimensión social, en la que se reflejan aspectos relacionados con las posibilidades de interacción normalizada con la sociedad de acogida; y, en tercer lugar, una dimensión político cívica, en la que se incluye información en relación con su participación activa en la toma de decisiones públicas de la sociedad de acogida.

Las hipótesis que se trabajan en relación con la influencia de la situación de integración en el uso de los servicios, asumen que, en general, una mayor integración redundará en menor uso de servicios específicos, pero especialmente en cuanto a su dimensión económica, pues una mejor situación económica debería redundar en una menor necesidad de servicios tradicionalmente asociados a la satisfacción de necesidades básicas, entre los que se incluyen buena parte de los servicios de carácter comunitario.

Por su parte, las oportunidades de contacto y participación con la sociedad de acogida, reflejadas en los Indicadores de Integración Social y Político Cívica, redundarán en un mayor conocimiento y uso de los servicios públicos, especialmente aquellos de carácter comunitario, por suponer una mayor relación con el entorno mayores oportunidades para acceder a servicios al alcance de todos los individuos, con independencia de su origen geográfico.

La Tabla 2 muestra los ítems incluidos en cada uno de los Índices Sintéticos de Integración elaborados para cada una de las dimensiones, calculados a partir de la media de los ítems incluidos en cada dimensión.

Tabla 2. Subculturas, modelo de barrio y situación de integración. Operacionalización de conceptos

\begin{tabular}{|c|c|c|}
\hline Concepto & Dimensión & Subdimensión \\
\hline \multirow{2}{*}{$\begin{array}{c}\text { Contexto } \\
\text { (Modelo de barrio) }\end{array}$} & Presencia & $\%$ población inmigrante en el barrio \\
\hline & Heterogeneidad & Diversidad de nacionalidades en el barrio \\
\hline \multirow{8}{*}{$\begin{array}{l}\text { Situación de integra- } \\
\text { ción }\end{array}$} & \multirow{3}{*}{ Económica } & Ingresos del individuo \\
\hline & & Situación profesional \\
\hline & & Ingresos de la unidad familiar \\
\hline & \multirow{3}{*}{ Social } & Años en España \\
\hline & & $\begin{array}{l}\text { Volumen de la red de relaciones en la sociedad de acogida } \\
\text { (inmigrantes y no inmigrantes) }\end{array}$ \\
\hline & & Estabilidad administrativa \\
\hline & \multirow{2}{*}{ Política } & Participación en elecciones \\
\hline & & Participación en entidades sociales \\
\hline \multirow{2}{*}{$\begin{array}{l}\text { Uso de los servicios } \\
\text { públicos }\end{array}$} & Servicios generales & Intensidad de uso de los servicios generales \\
\hline & Servicios específicos & Intensidad de uso de los servicios específicos \\
\hline \multirow{2}{*}{$\begin{array}{c}\text { Conocimiento } \\
\text { servicios públicos }\end{array}$} & Servicios generales & Conocimiento sobre la existencia de los servicios generales \\
\hline & Servicios & $\begin{array}{l}\text { Conocimiento sobre la existencia de los servicios específi- } \\
\text { cos }\end{array}$ \\
\hline
\end{tabular}

Fuente: Elaboración propia. 


\section{Fuentes de información utilizadas.}

La información utilizada para el análisis ha procedido de un cuestionario administrado entre personas inmigrantes mayores de 18 años y residentes en la ciudad de Sevilla. Dicho cuestionario se administró entre los meses de Agosto y Noviembre de 2009. El número total de cuestionarios analizados son 2.274, lo cual supuso un 1,98\% de error con respecto al universo sobre el que se diseñó la muestra (29.954), que era el total de población inmigrante residente en Sevilla a Enero de 2008. A continuación se ofrece la ficha técnica del cuestionario.

Tabla 3. Ficha técnica de la encuesta

\begin{tabular}{|l|c|}
\hline \multicolumn{1}{|c|}{ Centro responsable } & Centro de Sociología y Políticas Locales (UPO) \\
\hline Ámbito geográfico & Ciudad de Sevilla \\
\hline Universo de análisis & $\begin{array}{c}\text { Población inmigrante residente en la ciudad de Sevilla, } \\
\text { último dato publicado en el INE para el año 2008 (N=29.954) }\end{array}$ \\
\hline Tamaño de la muestra & (n) 2.274 \\
\hline Error máximo para datos globales & $1,98 \%$ \\
\hline $\begin{array}{l}\text { Procedimiento de recogida de la } \\
\text { información }\end{array}$ & Realización de encuestas personales domiciliarias \\
\hline Ámbito temporal de referencia & El trabajo de campo se realiza entre agosto y noviembre de 2009 \\
\hline
\end{tabular}

Fuente: Elaboración propia.

\section{La población inmigrante de Sevilla. Perfiles y lógicas de localización.}

En la ciudad de Sevilla la evolución de la población inmigrante ha sido de carácter ascendente, especialmente desde el año 2003 al año 2006, donde alcanzó su mayor crecimiento. Actualmente, se sitúa en torno al $4 \%$ respecto al total. Es importante destacar que dicha ciudad constituye un foco de atracción de población procedente de países en vías de desarrollo. Observando el Gráfico 1 se comprueba como en Sevilla, al igual que en el resto de España, existe una tendencia hacia la "latinoamericanización” del colectivo extracomunitario, la cual se ha ido afianzando en los últimos años. Entre los latinoamericanos tienen una mayor presencia de ciudadanos procedentes de países andinos y de América del Sur. Con respecto a la primera, y más numerosa, se debe destacar Ecuador como el país con mayor presencia en Sevilla. Junto a ellos se localizan los ciudadanos procedentes del Magreb y los procedentes de los países del este de Europa. Ello pone de manifiesto el carácter económico de la población inmigrante de Sevilla, cuya población provendría fundamentalmente de países en vías de desarrollo, lo que vendría a poner de manifiesto las razones que subyacen a la decisión de establecerse en Sevilla, como son fundamentalmente relacionadas con la búsqueda de un mayor bienestar económico en relación con sus países de origen. 
Gráfico 1. Población inmigrante por origen geográfico en Sevilla.

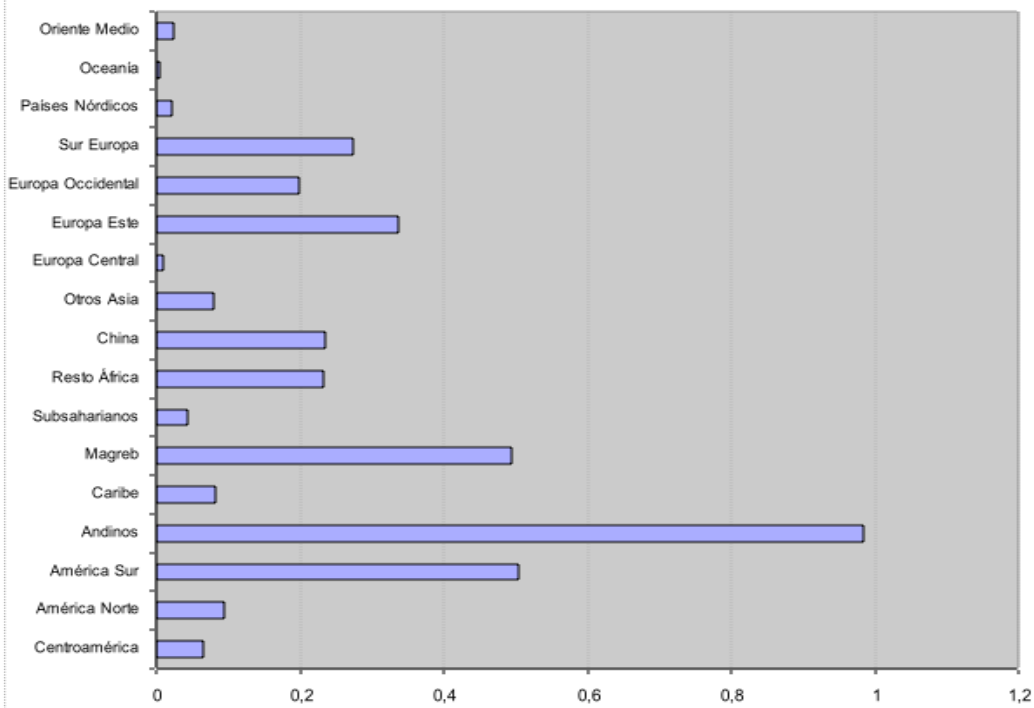

Fuente: Boletín Sociodemográfico de Sevilla (2006).

En este sentido, y tal como cabría esperar, los individuos que deciden residir en Sevilla lo hacen buscando oportunidades relacionadas con una mejor calidad de vida, representada en oportunidades de empleo, reencontrarse con amigos y familiares y, en tercer lugar, hacer uso de equipamientos y servicios adaptados a sus necesidades4. Ello se pone de manifiesto en las respuestas de los individuos encuestados en relación con los motivos de llegada a la ciudad de Sevilla, que se muestran en la Tabla 4.

Tabla 4. Razones de llegada a Sevilla

\begin{tabular}{|l|c|c|c|}
\hline \multirow{2}{*}{} & Sí & No & \multirow{2}{*}{$\begin{array}{c}\text { Total } \\
\text { de respuestas }\end{array}$} \\
\cline { 2 - 3 } & $\begin{array}{c}\text { \% sobre total } \\
\text { respuestas }\end{array}$ & \\
\hline Equipamientos y servicios adaptados a mis necesidades & 26,4 & 73,6 & 2.274 \\
\hline Posibilidades de prosperar económicamente & 61 & 38,9 & 2.274 \\
\hline Mayores posibilidades de encontrar una vivienda digna & 22,7 & 77,3 & 2.274 \\
\hline Mayor participación política y ciudadana & 9,2 & 90,8 & 2.274 \\
\hline Reencontrarse con familiares o amigos que ya se encontraban en Sevilla & 61,2 & 38,8 & 2.274 \\
\hline Oferta educativa/formativa adaptada a mis necesidades & 21 & 79 & 2.274 \\
\hline Otras & 10 & 90 & 2.273 \\
\hline
\end{tabular}

Fuente: Datos procedentes de la encuesta administrada entre la población inmigrante. 
Si bien podríamos decir que la población inmigrante residente en la ciudad de Sevilla presentaría unas características concretas en atención a su origen y razones de llegada a la ciudad, la pregunta que nos planteamos es si ello también pudiera relajarse en el uso y conocimiento de los servicios municipales. A ello se destina la siguiente sección.

\section{Patrones de interacción con los servicios públicos locales de las personas inmigrantes en la ciudad de Sevilla: sobre uso y conocimiento.}

\subsection{Análisis de los patrones de uso de los servicios.}

Esta sección está destinada al análisis de los patrones de uso de los servicios municipales por parte de la población inmigrante. En relación a este asunto, es importante conocer la forma en que las personas inmigrantes residentes en Sevilla hacen uso de los servicios municipales, pues, tal como señala Mora (2007: 26-27): "El análisis acerca del acceso, utilización y eficacia de los servicios públicos por parte de la población inmigrante, remite a una cuestión directamente relacionada con la garantía de los derechos sociales". Pero, tal y como señala el propio autor: "Las personas inmigrantes conforman un grupo que, generalmente, accede en menor medida al ejercicio de estos derechos, debilitados también para la población autóctona en un contexto de crisis de legitimidad de los Estados de Bienestar".

En coherencia con lo afirmado por Mora, se pone en evidencia, a partir de los análisis realizados, un bajo uso por parte de las personas inmigrantes de los servicios municipales sobre los que se consulta, si bien son más utilizados los servicios de carácter comunitario. La siguiente tabla muestra el uso de los servicios parte de la población inmigrante en una escala de 1 a 100 donde valores más cercanos a 1 mostrarían un bajo uso de dichos servicios y valores cercanos a 100 un muy alto uso de los mismos.

Tabla 5. Uso de los servicios: Estadísticos básicos

\begin{tabular}{|l|c|c|c|}
\hline \multirow{2}{*}{} & \multicolumn{2}{|c|}{$\mathrm{N}$} & \multirow{2}{*}{ Media } \\
\cline { 2 - 3 } & Válidos & Perdidos & \\
\hline Uso servicios específicos & 2274 & 0 & 7,7690 \\
\hline Uso servicios comunitarios & 2274 & 0 & 11,9437 \\
\hline Media general & 2274 & 0 & 9,8563 \\
\hline
\end{tabular}

Fuente: Datos procedentes de la encuesta administrada entre la población inmigrante.

Tal como se ha señalado anteriormente, los individuos inmigrantes hacen uso de servicios de carácter comunitario en mayor medida que los de carácter específico. Ahora bien, en coherencia con la primera de las proposiciones, relacionada con la 
existencia de diferencias en el uso y conocimiento de los servicios municipales en función el barrio de residencia, los análisis de Anova de un factor pondrían en evidencia la capacidad de explicación del barrio de residencia en los niveles de uso de los servicios, especialmente en relación con el uso de los servicios comunitarios, tal como se muestra en la Tabla 6.

Tabla 6. Uso de servicios con barrio de residencia como factor

\begin{tabular}{|l|l|c|c|c|c|c|}
\hline \multicolumn{2}{|c|}{} & Suma de cuadrados & gl & Media cuadrática & F & Sig. \\
\hline \multirow{4}{*}{$\begin{array}{l}\text { Uso servicios } \\
\text { específicos }\end{array}$} & Inter-grupos & 2661,238 & 2 & 1330,619 & 5,411 &, 005 \\
\cline { 2 - 7 } & Intra-grupos & 558420,068 & 2271 & 245,892 & & \\
\cline { 2 - 8 } & Total & 561081,306 & 2273 & & & \\
\hline \multirow{2}{*}{$\begin{array}{l}\text { Uso servicios } \\
\text { comunitarios }\end{array}$} & Inter-grupos & 28252,856 & 2 & 14126,428 & 56,483 &, 000 \\
\cline { 2 - 8 } & Intra-grupos & 567978,162 & 2271 & 250,100 & & \\
\cline { 2 - 8 } & Total & 596231,017 & 2273 & & & \\
\hline
\end{tabular}

Fuente: Datos procedentes de la encuesta administrada entre la población inmigrante.

Junto a ello, a la luz de los datos analizados y reflejados en la Tabla 7, una mayor presencia de la población inmigrante en el barrio de residencia redundaría en un mayor uso de servicios comunitarios, en coherencia con la proposición 2. Esto es, en el uso y conocimiento de los servicios jugaría un papel importante el efecto de las oportunidades de interacción entre los individuos, que podría estar facilitando el acceso a la información sobre cómo acceder y utilizar estos servicios.

Finalmente, los análisis de comparación de medias mostrados en la Tabla 7 vendrían a confirmar la proposición 3, pues mientras que los barrios en auge y multiculturales mostrarían un mayor uso de servicios específicos, los barrios autóctonos serían los que en menor medida harían uso de ellos, siendo más intensivo en estos últimos el uso de servicios comunitarios. En definitiva, los barrios en auge y multiculturales mostrarían patrones de uso más propios de la población inmigrante, mientras que los barrios autóctonos mostrarían patrones más similares a los de la población autóctona, haciendo uso mayor de servicios comunitarios, caracterizados por destinarse a la población en su conjunto, con independencia de su origen.

Hay que destacar que en principio podría pensarse que el elemento territorial, la cercanía física de los ciudadanos a los servicios podría ser la explicación, pero los servicios analizados se localizan por igual en todas las zonas de la ciudad, a excepción de los de carácter específico que se localizan en el zona norte y centro de la ciudad, donde se daría una mayor cercanía por parte de los barrios multiculturales, localizados en el distrito Macarena. Ahora bien, esa lógica no funcionaría para los servicios comunitarios, donde también se reconoce un mayor conocimiento de los servicios en esta tipología de barrios. 
Tabla 7. Uso de servicios en función del modelo de barrio

\begin{tabular}{|c|c|c|c|c|}
\hline \multicolumn{2}{|c|}{ MODELOS_BARRIO_GRAL } & $\begin{array}{l}\text { Servicios } \\
\text { específicos }\end{array}$ & $\begin{array}{c}\text { Servicios } \\
\text { comunitarios }\end{array}$ & Diferencia \\
\hline \multirow[t]{3}{*}{ AUGE } & Media & 8,6702 & 9,0618 & \multirow{3}{*}{$-0,3916$} \\
\hline & $\mathrm{N}$ & 1311 & 1311 & \\
\hline & Desv. típ. & 16,28842 & 13,90600 & \\
\hline \multirow[t]{3}{*}{ MULTICULTURAL } & Media & 6,7513 & 16,7380 & \multirow{3}{*}{$-9,9867$} \\
\hline & $\mathrm{N}$ & 748 & 748 & \\
\hline & Desv. típ. & 14,96514 & 18,82289 & \\
\hline \multirow[t]{3}{*}{ AUTÓCTONO } & Media & 5,8140 & 12,8372 & \multirow{3}{*}{$-7,0232$} \\
\hline & $\mathrm{N}$ & 215 & 215 & \\
\hline & Desv. típ. & 14,26811 & 15,28415 & \\
\hline \multirow[t]{3}{*}{ Total } & Media & 7,7690 & 11,9437 & \multirow{3}{*}{$-4,1747$} \\
\hline & $\mathrm{N}$ & 2274 & 2274 & \\
\hline & Desv. típ. & 15,71134 & 16,19599 & \\
\hline
\end{tabular}

Fuente: Datos procedentes de la encuesta administrada entre la población inmigrante.

Ahora bien, ¿es solamente el territorio o contexto en el que reside la población inmigrante lo que establece diferencias en el uso de los servicios, o podrían también mostrarse diferencias en función de la situación de integración de los individuos?

Tal como se muestra en la siguiente tabla, la integración, en su dimensión social, referida a aspectos relacionados con una relación normalizada con su entorno, redundaría en un menor uso servicios específicos, mientras que lo haría en un sentido contrario en los de carácter comunitario. Ello pone de manifiesto que una mayor interacción con el entorno y experiencia en el país de destino facilitarían el acceso a servicios comunitarios, posiblemente como resultado de mayores recursos para el acceso a los servicios, en términos de redes de conocimiento a través de mayor interacción con la sociedad de acogida. En ello, sin duda, estaría jugando además un papel relevante la situación administrativa del individuo, pues, tal como señalan aportaciones como las de Mora (2007), se muestra un acceso diferencial a los servicios en función de la situación administrativa en la que se encuentre el individuo.

Por el contrario, la situación de integración, en su dimensión económica, redundaría en un menor uso de servicios comunitarios y un mayor uso de servicios específicos. En este sentido, servicios como el foro de participación de las personas inmigrantes, incluido entre los servicios de carácter específico, son mecanismos de participación no relacionados con necesidades de carácter básico. Por ello, es lógico pensar que a medida que la persona disfrute de una mejor situación económica dependerá en menor medida de los servicios públicos que le satisfagan necesidades básicas y, en consecuencia, podría destinar más tiempo a hacer uso de otros, por 
ejemplo, mecanismos de participación ciudadana como el foro de participación de las personas inmigrantes.

Finalmente, la situación de integración, en la dimensión cívico política, daría como resultado un menor uso de servicios de carácter específico y un mayor uso de servicios de carácter comunitario, si bien la relación estadística en ambos casos es considerablemente baja. Aun así, los análisis estarían mostrando que una mayor participación en la toma de decisiones de la ciudad que daría como resultado un mayor uso de los servicios que se encuentran al alcance de toda la ciudadanía, con independencia de su origen geográfico. En este sentido, quizá una mayor participación en las decisiones de la ciudad sería también reflejo de un mayor auto concepto de ciudadano y, por tanto, un mayor uso de servicios destinados a la ciudadanía en su conjunto.

Tabla 8. Uso de los servicios en función de la situación de integración

\begin{tabular}{|c|c|c|c|}
\hline & & $\begin{array}{c}\text { Servicios } \\
\text { específicos } \\
\end{array}$ & $\begin{array}{c}\text { Servicios } \\
\text { comunitarios } \\
\end{array}$ \\
\hline \multirow{3}{*}{ ISI_ECONÓMICO } & Correlación de Pearson &, $097^{*}$ &,$- 226^{* *}$ \\
\hline & Sig. (bilateral) & ,025 & ,000 \\
\hline & $\mathrm{N}$ & 537 & 537 \\
\hline \multirow{3}{*}{ ISI_CÍVICO/POLÍTICO } & Correlación de Pearson &,$- 067^{*}$ & ,060 \\
\hline & Sig. (bilateral) & ,035 & ,060 \\
\hline & $\mathrm{N}$ & 993 & 993 \\
\hline \multirow{3}{*}{ ISI_SOCIAL } & Correlación de Pearson &,$- 126^{*}$ & $273^{* *}$ \\
\hline & Sig. (bilateral) & ,018 & 000 \\
\hline & $\mathrm{N}$ & 349 & 349 \\
\hline
\end{tabular}

Fuente: Datos procedentes de la encuesta administrada entre la población inmigrante.

En definitiva, los análisis realizados han confirmado las hipótesis planteadas en relación con la importancia del modelo de barrio, conformado a partir de la presencia y diversidad de la población inmigrante, como elemento diferenciador en los patrones de uso de los servicios municipales por parte de la población inmigrante. A su vez, la situación de integración de los individuos también sería un elemento a tener en cuenta, pues en atención a su situación de integración en cada una de las dimensiones analizadas (económica, social y cívica), se establecerían diferencias en relación con el uso de los servicios específicos y comunitarios. Ahora bien, del análisis de las diferentes dimensiones que conforman el concepto de integración se desprende que cada una de ellas se relaciona con el uso de los servicios de forma diferente, por lo que, tal como se pone de manifiesto en la literatura el respecto, la 
integración social (también para el análisis de las personas inmigrantes) no ha de considerarse como un concepto unitario, sino que cada una de las dimensiones que lo conforman (económica, social y cívico-política) mostraría tendencias diferentes en su relación con el uso servicios municipales de carácter comunitario y específico.

\subsection{Conocimiento de los servicios municipales}

Esta sección se destina el análisis del conocimiento de los servicios municipales por parte de la población inmigrante. De la misma forma que para el uso de los servicios municipales, esta sección analiza el conocimiento, tanto sobre servicios de carácter específico como de carácter comunitario, atendiendo al barrio de residencia, así como la situación de integración del individuo como variables diferenciadoras con la intensidad del uso y conocimiento de los servicios por parte de dicha población.

En primer lugar, los análisis de comparación de medias muestran que, en general, las personas inmigrantes tienen un conocimiento medio-bajo de los servicios municipales, si bien, es superior el de los servicios de carácter comunitario frente a los servicios de carácter específico, tal como se muestra en la Tabla 9.

Tabla 9. Conocimiento de los servicios: estadísticos básicos

\begin{tabular}{|l|c|c|c|}
\hline \multirow{2}{*}{} & \multicolumn{2}{|c|}{ N } & \multirow{2}{*}{ Media } \\
\cline { 2 - 3 } & Válidos & Perdidos & Med \\
\hline Conocimiento servicios específicos & 1699 & 575 & 16,3626 \\
\hline Conocimiento servicios comunitarios & 2048 & 226 & 25,1161 \\
\hline
\end{tabular}

Fuente: Datos procedentes de la encuesta administrada entre la población inmigrante.

Ahora bien, se muestran diferencias sensibles entre los barrios de residencia. Tal como se planteaba en la proposición 1 , se pone de manifiesto en el análisis de Anova de un factor que el barrio de residencia podría introducir diferencias en los niveles de conocimiento que las personas inmigrantes tiene respecto de los servicios sobre los que se consulta y, de forma especial, sobre los servicios de carácter comunitario. 
Tabla 10. Modelo de Barrio

\begin{tabular}{|c|l|c|c|c|c|c|}
\hline \multicolumn{2}{|c|}{} & $\begin{array}{c}\text { Suma de } \\
\text { cuadrados }\end{array}$ & $\mathrm{gl}$ & $\begin{array}{c}\text { Media } \\
\text { cuadrática }\end{array}$ & F & Sig. \\
\hline \multirow{4}{*}{$\begin{array}{c}\text { Conocimiento servicios } \\
\text { específicos }\end{array}$} & Inter-grupos & 2294,136 & 2 & 1147,068 & 2,324 &, 098 \\
\cline { 2 - 8 } & Intra-grupos & 837270,967 & 1696 & 493,674 & & \\
\cline { 2 - 8 } & Total & 839565,104 & 1698 & & & \\
\hline \multirow{3}{*}{$\begin{array}{c}\text { Conocimiento servicios } \\
\text { comunitarios }\end{array}$} & Inter-grupos & 68012,477 & 2 & 34006,238 & 59,425 &, 000 \\
\cline { 2 - 8 } & Intra-grupos & 1170270,410 & 2045 & 572,259 & & \\
\cline { 2 - 8 } & Total & 1238282,887 & 2047 & & & \\
\hline
\end{tabular}

Fuente: Datos procedentes de la encuesta administrada entre la población inmigrante.

Además, en coherencia con la segunda de las proposiciones planteadas, la presencia masiva de personas inmigrantes en el barrio, así como las posibilidades de interacción con individuos de diversas nacionalidades, situación reflejada en los barrios incluidos en el modelo multicultural, daría como resultado un mayor conocimiento de los servicios municipales, tal como se muestra en la Tabla 11. Es decir, la coincidencia en el espacio de personas inmigrantes, esto es, mayor presencia debería redundar en un mayor conocimiento de los servicios locales, pues existirían mayores posibilidades de acceso a los mismos a través de facilitarse información entre unos individuos y otros en relación con su existencia y formas de uso.

Por su parte, una menor presencia y diversidad de la población inmigrante daría como resultado un menor conocimiento de los servicios de carácter específico, tal como sucede en los barrios autóctonos, mostrando de esta formas unos patrones de uso más similares a los de la población autóctona. Por el contrario, en los barrios en auge, con mayor presencia de la población inmigrante, se daría un menor conocimiento de servicios de carácter comunitario, posiblemente y en coherencia con las hipótesis planteadas en la proposición 3, mostrando dicha población patrones de uso más propios de la población inmigrante. Dicho de otra forma, las diferencias fundamentales en el conocimiento de los servicios municipales por parte de la población inmigrante se encontrarían entre los barrios autóctonos, como efecto de la presencia masiva de población autóctona, respecto a los barrios en auge, como efecto de la presencia masiva de población inmigrante, siendo mayor el conocimiento de servicios comunitarios, destinados a la ciudadanía en su conjunto, en los barrios de carácter autóctono, frente a los barrios en auge, en los que sucedería lo contrario, es decir, un mayor conocimiento de servicios destinados específicamente a la población inmigrante. 
Tabla 11. Conocimiento de los servicios en función del modelo de barrio

\begin{tabular}{|l|l|c|c|}
\hline \multicolumn{2}{|l|}{ MODELOS_BARRIO_GRAL } & $\begin{array}{c}\text { Conocimiento } \\
\text { servicios específicos }\end{array}$ & $\begin{array}{c}\text { Conocimiento } \\
\text { servicios comunitarios }\end{array}$ \\
\hline \multirow{4}{*}{ AUGE } & Media & 16,2713 & 20,6360 \\
\cline { 2 - 4 } & $\mathrm{N}$ & 1054 & 1167 \\
\cline { 2 - 4 } & Desv. típ. & 22,15331 & 22,76565 \\
\hline \multirow{3}{*}{ MULTICULTURAL } & Media & 17,5983 & 33,0318 \\
\cline { 2 - 4 } & $\mathrm{N}$ & 483 & 702 \\
\cline { 2 - 4 } & Desv. típ. & 21,86407 & 25,78967 \\
\hline \multirow{2}{*}{ AUTÓCTONO } & Media & 13,2716 & 23,2806 \\
\cline { 2 - 5 } & $\mathrm{N}$ & 162 & 179 \\
\cline { 2 - 5 } & Desv. típ. & 23,65345 & 23,66931 \\
\hline \multirow{2}{*}{ Total } & Media & 16,3626 & 25,1161 \\
\cline { 2 - 5 } & $\mathrm{N}$ & 1699 & 2048 \\
\cline { 2 - 5 } & Desv. típ. & 22,23609 & 24,59524 \\
\hline
\end{tabular}

Fuente: Datos procedentes de la encuesta administrada entre la población inmigrante.

Si bien las diferencias en función del modelo de barrio podrían estar poniendo de manifiesto la existencia de subculturas propias en relación con el uso y conocimiento de los servicios, la situación de integración del individuo en la sociedad de acogida también podría relacionarse con los diferentes niveles de conocimiento de los servicios municipales, tal como sucediera en el uso de los mismos.

En este sentido, en coherencia con las hipótesis planteadas, una mejor situación económica se relacionaría con un menor conocimiento sobre los servicios comunitarios. Tal como sucediera en relación con el uso de los servicios, pareciera que una mejor situación económica del individuo estaría reflejando, como es lógico, una menor necesidad de servicios destinados a proveer al ciudadano de bienes de carácter básico, por lo que ello redundaría en un menor conocimiento y uso de los mismos.

Por su parte, la participación cívico política se relaciona con un mayor conocimiento de los servicios, tanto de carácter específico como de carácter general, con independencia del uso que se hagan de ellos, tal como se ha puesto de manifiesto en la sección anterior. A la luz de los resultados obtenidos, pareciera que una participación activa en la sociedad de acogida, redundaría en un conocimiento mayor de la 
totalidad de servicios a disposición del ciudadano, con independencia de que finalmente sean utilizarlos por parte de la población analizada.

Finalmente, mayor interacción normalizada con la sociedad de acogida o, dicho de otra forma, la situación de integración en cuanto a su dimensión social, daría como resultado un mayor conocimiento de servicios de carácter comunitario, de la misma forma que sucediera en relación con el uso de los mismos.

Tabla 12. Conocimiento de los servicios en función de la situación de integración

\begin{tabular}{|c|l|c|c|}
\hline \multicolumn{2}{|c|}{} & $\begin{array}{c}\text { Conocimiento } \\
\text { servicios específicos }\end{array}$ & $\begin{array}{c}\text { Conocimiento } \\
\text { servicios comunitarios }\end{array}$ \\
\hline \multirow{4}{*}{ ISI_ECONÓMICO } & Correlación de Pearson &, 083 &,$- 178^{* *}$ \\
\cline { 2 - 4 } & Sig. (bilateral) &, 154 &, 000 \\
\cline { 2 - 4 } & $\mathrm{N}$ & 300 & 466 \\
\hline \multirow{3}{*}{ ISI_CIVICO/POLÍTICO } & Correlación de Pearson &, $113^{* *}$ &, $187^{* *}$ \\
\cline { 2 - 4 } & Sig. (bilateral) &, 004 &, 000 \\
\cline { 2 - 4 } & $\mathrm{N}$ & 669 & 946 \\
\hline \multirow{3}{*}{ ISI_SOCIAL } & Correlación de Pearson &,- 035 &, $423^{* *}$ \\
\cline { 2 - 4 } & Sig. (bilateral) &, 581 & 323 \\
\cline { 2 - 4 } & $\mathrm{N}$ & 252 & \\
\hline \multirow{2}{*}{$\begin{array}{l}\text { **. La correlación es significativa al nivel 0,01 (bilateral). } \\
\text { *. La correlación es significante al nivel 0,05 (bilateral). }\end{array}$} \\
\hline
\end{tabular}

Fuente: Datos procedentes de la encuesta administrada entre la población inmigrante.

En definitiva, tal como se ha puesto de manifiesto en los análisis realizados, la situación de integración del individuo se pondría en relación con distintos niveles de conocimiento de los servicios, atendiendo a las dimensiones consideradas. De esta forma, encontrarse en una situación económicamente estable se pondría en relación con un menor conocimiento de los servicios comunitarios, mientras que las otras dos dimensiones consideradas (cívico-política y social) lo harían en sentido contrario, esto es, a medida que el individuo tenga mayor participación a interacción en la sociedad de acogida mayor sería su grado de conocimiento sobre los servicios, en especial aquellos de carácter comunitario.

\section{Conclusiones}

Este artículo se ha destinado a analizar los patrones de interacción de la población inmigrante con el sector público, a través de dos cuestiones concretas, como son, el conocimiento y uso de servicios públicos en la ciudad de destino. Dichos patrones 
se han estudiado atendiendo al barrio de residencia, así como la situación de integración del individuo.

Tal como se ha puesto de manifiesto a lo largo del trabajo, a través de las propuestas existentes en la sociología urbana, concretamente las propuestas enmarcadas en la ecología urbana, el barrio de residencia podría constituir un espacio para el surgimiento de subculturas propias, entendidas éstas en términos de la relación de los individuos con los servicios públicos locales. De esta forma, las subculturas propias se han analizado en atención a tres proposiciones específicas. En primer lugar, la existencia de patrones de uso y conocimiento de los servicios locales diferentes en función del modelo de barrio. En segundo lugar, dichas diferencias se dan como consecuencia de las oportunidades de contacto de los individuos. Finalmente, la existencia de efectos de difusión entre los grupos mayoritarios hacia los minoritarios en los diferentes barrios.

De esta forma, los análisis realizados han mostrado no solamente la existencia de diferentes patrones de uso y conocimiento de los servicios locales en función del barrio de residencia, en coherencia con la primera de las proposiciones planteadas en la que se hacía referencia a la capacidad del barrio para introducir diferencias en los patrones de interacción de la población inmigrante con los servicios públicos. Además, se ha mostrado que una mayor presencia y diversidad de la población inmigrante daría como resultado un mayor uso y conocimiento de los servicios locales. Dicha relación se ha puesto en relación con mayores oportunidades de contacto de los individuos y con ello mayor acceso a la información necesaria para el conocimiento sobre su existencia y uso de los mismos.

Finalmente la tercera de las proposiciones planteadas, proponía la existencia de efectos de difusión entre la población autóctona e inmigrante en el uso y conocimiento de los servicios. En este sentido, los análisis han mostrado patrones de uso y conocimiento más similares a los de la población autóctona en barrios en los que la población inmigrante sería minoría y más propios del colectivo inmigrante en barrios donde la población inmigrante tendría una presencia significativa.

Junto a la consideración de elementos de carácter territorial, este trabajo ha analizado la situación de integración del individuo como elemento diferenciador en el tipo de uso y conocimiento de los servicios públicos locales. En este sentido, se ha puesto en evidencia que cada una de las dimensiones de la situación de integración de los individuos consideradas, y en coherencia con la literatura existente, se ponen en relación con diferentes niveles de uso y conocimiento de los servicios públicos locales.

De esta forma, tal como cabría esperar, la situación integración relacionada con la dimensión económica redundaría en un menor uso y conocimiento de los servicios comunitarios, asociados fundamentalmente con el acceso a servicios de carácter básico, mientras que lo haría en sentido contrario si atendemos a los servicios de carácter específico. Por su parte, una mayor integración en las dimensiones cívica y social, esto es, una interacción normalizada con el entorno redundaría en un mayor uso y conocimiento de servicios de carácter comunitario y menos de carácter específico. 
A tenor de estos resultados, este trabajo ha puesto de manifiesto que la situación de integración en la sociedad de acogida del individuo ha de considerarse a la hora de conocer el tipo de relación que establece con los servicios públicos. Ahora bien, su capacidad explicativa no puede entenderse como un todo, sino que las diferentes dimensiones de las que se compone (económica, social y político-cívica) estarían actuando de forma distinta en su relación con el conocimiento y uso de los servicios públicos por parte de la población objeto de estudio.

En definitiva, lo que este artículo ha tratado de poner de manifiesto es que la condición de inmigrante no constituye un atributo en el individuo a partir del cual conocer o incluso predecir el tipo de relación que se establece con los servicios públicos, más concretamente, sus patrones de conocimiento y uso de los mismos. Más bien al contrario, existirían una serie de condicionantes a partir de los cuales dicha relación se daría de forma diferente en dicha población, para el caso que nos ocupa, el ámbito o contexto territorial de residencia, concretamente el barrio, así como la situación de integración en el lugar de acogida. Ambos constituirían aspectos a partir de los cuales comprender diferentes patrones de interacción de dicha población con los servicios públicos.

\section{Referencias bibliográficas}

Área de Estadística del Ayuntamiento de Sevilla (2006): Boletín Sociodemográfico de Sevilla (2006). Ayuntamiento de Sevilla.

Bevelander, P. y Pendakur, R. (2008): Electoral participation as a measure of social exclusion for natives, inmigrants and descendants in Sweden. IZA Discussion paper, 3764.

Buchanam, J. (1971): “Principles of Urban Strategy”, en Public Choice. 4, pp.1-16.

Castles, M y Miller, M.(1993): The age of migration: international population movements in the moderns World. Londres: MacMillan.

Commins, P. (comp) (1993): Combating exclusión in Ireland 1990-1994. A midway report. European Commission, Brussels.

Esping Andersen, G. (2009): Los tres grandes retos del Estado del Bienestar. Ariel: Barcelona.

Fisher, C.S. 1975. “Toward a subcultural theory of urbanism”. American Journal of Sociology 80. (May): 1319-41.

Fisher, C.S. 1995. "The subcultural theory of urbanism: a twentieth- year assesment”. The American Journal of Sociology. Vol. 101. No. 3 (Nov. 1995). pp. 543-577.

Hidalgo, Alfredo (1985): "El marco jurídico administrativo de los servicios sociales de la Comunidad Autónoma Andaluza”, en Alternativas. Cuadernos de Trabajo Social. N. 4 
Huete, M.A.; Muñoz, R.; Navarro, C.; Rodríguez, M.J.: (2011): Diagnóstico de la población inmigrante en la ciudad de Sevilla. Ayuntamiento de Sevilla

(en prensa).

Huete, M.A. y Muñoz, R. (2011): Las lógicas de localización de la población inmigrante en Sevilla. El caso de la ciudad de Sevilla, en Skripta Nova (en prensa).

García Cívico, J.: "La medición de la integración social de los inmigrantes a través de un sistema coherente con la noción de ciudadanía inclusiva”, en Universitas. Revista de Filosofía, Derecho y Política. no 12, julio 2010.

González-Ferrer, A. (2010): "The electoral participation of naturalized inmigrants in ten European cities”, en L. Morales y M. Giugin (eds.), Social capital, political participation and migration in Europe. Making multicultural democracy work?. Basingtoke: Palgrave Macmillan.

Laparra, M. (2001): Una perspectiva de conjunto sobre el espacio social de exclusión. Probreza y exclusión: La "malla de seguridad” en España. Luis Moreno. CSIC Politeya. Madrid.

Moreno, F.J. y Bruquetas, M. (2011): Inmigración y Estado de bienestar en España. Colección Estudios Sociales. Núm. 31. Obra Social La Caixa.

Peterson, P.E. (1982): City limits. Chicago: Chicago University Press.

Rank, M.R. y Hirschl, T.A. (1988): "A rural-urban comparison of welfare exits: the importance of population density”, en Rural Sociology 53 (2), 1988, pp. 190-206.

Secretaría de Estado de Inmigración y Emigración del Ministerio de Trabajo y Asuntos Sociales (2010): Indicadores de integración de inmigrantes. Propuesta para la elaboración de un sistema de indicadores comunes para la integración social. Secretaría de Estado de Inmigración y Emigración del Ministerio de Trabajo y Asuntos Sociales.

Stein, M.R. (1990): Urban alternatives. Public and private markets in the provision of local services. Pittsburg: University of Pittsburgh Press.

VV.AA (2007): "Una propuesta de consenso sobre el concepto de exclusión. Implicaciones metodológicas”, en Revista Española del Tercer Sector. Numero 5. Enero-Abril.2007. 